\title{
37. ALGUNOS BRIÓFITOS INTERESANTES DE LA SIERRA DE INVERNADEIRO (ORENSE, NW DE ESPAÑA)
}

\author{
Juan REINOSO FRANCO y María del Carmen VIERA BENÍTEZ
}

Palabras clave. Briófitos, corología, NO. de España

Damos a conocer una serie de briófitos, en su mayor parte no citados en la flora de Orense, recolectados en la Sierra de Invernadeiro. La zona de estudio, por otra parte totalmente inédita desde el punto de vista briológico, integra un gran macizo montañoso que se encuentra en la zona interior de Galicia y constituye un territorio híbrido de vegetación caducifolia y perennifolia situado en pleno corazón de la provincia.

Geológicamente los materiales están formados por areniscas, pelitas, esquistos y sobre todo pizarras del Ordovícico y Silúrico. De acuerdo con Carballeira et al. (1983) los datos climáticos correspondientes a la estación de Vilariño de Couso (municipio donde se ha centrado básicamente nuestro estudio ) son : temperatura media anual de $8,4{ }^{\circ} \mathrm{C}$, temperatura media de las máximas para el mes más cálido (Julio) de $24,4{ }^{\circ} \mathrm{C}$ y una temperatura media de las mínimas para el mes más frío (Enero) de $-1,3{ }^{\circ} \mathrm{C}$. El territorio soporta una fuerte continentalidad pluvial con precipitaciones que superan los $1300 \mathrm{~mm}$., con un máximo a mediados de invierno.

La vegetación superior del territorio atlántico corresponde a la serie Vaccinio myrtilli-Querceto roboris sigmetum (robledales montanos) y tan solo en las áreas más altas se establece la serie Luzulo henriquesii-Betuleto celtibericae sigmetum (abedulares). En territorio mediterráneo la vegetación potencial corresponde a la serie Holco molli-Querceto pyrenaicae sigmetum (melojares húmedos).

Los táxones que constituyen novedad para la provincia de Orense se señalan con un asterisco. El material estudiado se encuentra depositado en el Herbario SANTBryo. (Universidad de Santiago).

\section{CATÁLOGO}

Anisothecium palustre (Dicks. ) I. Hag. *

ORENSE: Sierra de Invernadeiro, Cabeza do Cernado, 29TPG4163, suelo encharcado, SANT-Bryo 2182. Taxon señalado por primera vez en Galicia por Reinoso(1984) en los bosques de Caaveiro; junto con la cita que ahora se aporta son las únicas referencias gallegas conocidas.

\section{Bartramia stricta Brid.}

ORENSE: Sierra de Invernadeiro, fraga da Pena, 29TPG4167, rocas húmedas, SANTBryo 2183. Las cercanías de la capital y Sobradelo eran las únicas referencias orensanas conocidas para el taxon, Casares-Gil(1915) y Rungby(1964).

Bryum alpinum With.

ORENSE: Sierra de Invernadeiro, Corga Seca, 29TPG3869, rocas, SANT-Bryo 2184. Nueva localización orensana a añadir a la hasta ahora única conocida de Sobradelo Allorge(1929).

Bryum caespiticium Hedw. *

ORENSE: Sierra de Invernadeiro, Vegas de Meda 29TPG3766, rocas, SANT-Bryo 
2185. Las dos únicas referencias gallegas para el taxon se debían a Reinoso(1984) y García et al. (1989).

Dicranum scoparium Hedw. *

ORENSE: Sierra de Invernadeiro, Anxiñeira, 29TPG3661, terrícola en suelos de bosques, SANT-Bryo 2186. Desconocemos otra referencia bibliográfica para la provincia.

Diphyscium foliosum (Hedw. ) Mohr.

ORENSE: Sierra de Invernadeiro, Lombo de Misueira 29TPG3463, entre fisuras de rocas, SANT-Bryo 2187. Añádase esta nueva localidad orensana a la única hasta ahora conocida de Los Peares Allorge(1934).

Drepanocladus fluitans (Hedw. ) Warnst. *

ORENSE: Sierra de Invernadeiro, Cabeza do Cernado, 29TPG4163, arroyo, SANTBryo 2188. Ha sido escasamente herborizado en Galicia, solamente nos constan los testimonios de Casares-Gil(1915) y Allorge(1928).

Encalypta streptocarpa Hedw. *

ORENSE: Sierra de Invernadeiro, alto de Touza, 29TPG4062, muro, SANT-Bryo 2189. La primera cita gallega corresponde a una herborización efectuada en la Sierra del Caurel, Reinoso(1985). Segunda cita regional.

Eurhynchium praelongum (Hedw. )B. S. G.

ORENSE: Sierra de Invernadeiro, Lama, 29TPG4059, epífito sobre roble, SANTBryo 2190. Rungby(1964) señala la especie en las proximidades de la ciudad de Orense. Segunda cita provincial.

Fissidens curnowii Mitt.

ORENSE: Sierra de Invernadeiro, Fuxo de Can, 29TPG3564, talud, SANT-Bryo 2191. La única cita provincial se debe a Potier de la Varde(1945) que señala la planta en las proximidades de Piñor y Verín.

Fissidens incurvus Starke ex Röhl. *

ORENSE: Sierra de Invernadeiro, arroyo de Meda, 29TPG3766, tierra, SANT-Bryo 2192. Luisier(1918) cita el taxon en la localidad pontevedresa de Camposancos que con la cita orensana son las únicas referencias regionales.

Fissidens polyphyllus Wills.

ORENSE: Sierra de Invernadeiro, arroyo de Meda, 29TPG3766, tierra, SANT-Bryo 2193. Nueva localización orensana a añadir a la conocida de Piñor Allorge(1934). Segunda cita provincial.

Fissidens serrulatus Brid.

ORENSE: Sierra de Invernadeiro, arroyo de Meda, 29TPG3766, tierra, SANT-Bryo 2194. A pesar de ser un elemento frecuente en la brioflora gallega, hasta ahora no se había señalado el taxon en la provincia de Orense.

Grimmia ovalis (Hedw. ) Lindb. *

ORENSE: Sierra de Invernadeiro, 29TPG3865, Collado das Rubias, rocas, SANTBryo 2195. Representa una notable aportación para la brioflora de Galicia al ser los bosques de Caaveiro la única referencia conocida, Reinoso(1985).

Grimmia trichophylla Grev.

ORENSE: Sierra de Invernadeiro, Collado das Rubias, 29TPG3865, rocas, SANTBryo 2196. Nueva localización orensana a añadir a la inicial de Colmeiro(1889). Segunda cita provincial. 
Gymnostomum aeruginosum Sm. *

ORENSE: Sierra de Invernadeiro, Ribeira Pequena, 29TPG3764, muro, SANT-Bryo 2197. Taxon escasamente representado en la flora gallega, solamente nos constan las referencias de Merino(1916) y Rungby(1964) que lo señalan en las localidades pontevedresas de Camposancos, La Guardia, El Rosal y Porriño.

Homalothecium sericeum (Hedw. ) B. S. G.

ORENSE: Sierra de Invernadeiro, Val de Figueiro, 29TPG3668, muro, SANT-Bryo 2198. En la provincia sólo conocíamos las citas de Levier(1880) y Rungby(1964) señalando el taxon en las proximidades de la capital.

Neckera crispa Hedw.

ORENSE: Sierra de Invernadeiro, Rucín, 29TPG3768, rocas, SANT-Bryo 2199. La primera localidad gallega para el taxon fue Caaveiro, Reinoso(1984), que con la que ahora se aporta constituyen las únicas referencias conocidas para el taxon en Galicia.

Orthotrichum diaphanum Brid. *

ORENSE: Sierra de Invernadeiro, Rucín, 29TPG3768, roble, SANT-Bryo 2200. Desconocemos otra referencia bibliográfica para la provincia.

Oxystegus tenuirostris (Hook. ) Tayl. *

ORENSE: Sierra de Invernadeiro, Campa de Figueiro, 29TPG3668, esquistos, SANTBryo 2201. Los bosques de Caaveiro, una vez más, constituyen la otra localidad gallega para el taxon Reinoso(1984).

Plagiothecium nemorale (Mitt. )Jaeg. *

ORENSE: Sierra de Invernadeiro, arroyo de Cavaloiro, 29TPG3967, tierra húmeda, SANT-Bryo 2202.

Pogonatum aloides (Hedw. ) P. Beauv. *

ORENSE: Sierra de Invernadeiro, Lama, 29TPG4059, talud, SANT-Bryo 2203. A pesar de ser frecuentísimo el taxon en Galicia, no se había señalado en Orense.

Pogonatum nanum (Hedw. ) P. Beauv.

ORENSE: Sierra de Invernadeiro, Lama, 29TPG4059, talud, SANT-Bryo 2204. Solamente nos consta una referencia orensana debida a Casares-Gil(1915) que señala el taxon en las cercanías de la capital. Segunda cita provincial.

\section{Pohlia elongata Hedw. *}

ORENSE: Sierra de Invernadeiro, Ribeira Grande, 29TPG4065, suelo de bosque, SANT-Bryo 2205. Casares-Gil(1915) señala el taxon por primera vez en Galicia, concretamente en la localidad pontevedresa del valle de Cuntis. Reinoso(1984) la indica en Caaveiro. La localidad orensana constituye pues la tercera referencia regional.

Polytrichum juniperinum Hedw.

ORENSE: Sierra de Invernadeiro, Cabeza do Cernado, 29TPG4163, suelo de brezal, SANT-Bryo 2206. La única referencia del taxon en la provincia se debe a Levier(1880). Segunda cita provincial.

Polytrichum piliferum Hedw. *

ORENSE: Sierra de Invernadeiro, Cabeza do Cernado 29TPG4163, suelo de brezal, SANT-Bryo 2207. Aunque se trata de una planta muy frecuente en Galicia, no se conocía ninguna cita orensana.

Pterogonium gracile (Hedw. ) Sm.

ORENSE: Sierra de Invernadeiro, Corga Seca, 29TPG3869, epífito en roble, SANTBryo 2208. Especie escasamente citada en la flora orensana, las únicas referencias se deben a Colmeiro(1867) y a Levier(1880). 
Racomitrium heterostichum (Hedw. ) Brid.

ORENSE: Sierra de Invernadeiro, Lombo de Misueira, 29TPG3463, rocas, SANTBryo 2209. Añádase esta nueva localidad orensana a la inicial de Rungby(1964). Segunda cita provincial.

Racomitrium lanuginosum (Hedw. ) Brid. *

ORENSE: Sierra de Invernadeiro, Lombo de Misueira, 29TPG3463, rocas, SANTBryo 2210. A pesar de ser un elemento frecuente en Galicia se conocen pocas citas, en cualquier caso se trata la orensana de la estación más meridional; las anteriores de Luisier(1918), Allorge(1929), Rungby(1964), Casas(1972) y Reinoso(1984) corresponden a localidades costero-atlánticas.

Rhabdoweisia fugax (Hedw. )B. S. G.

ORENSE: Sierra de Invernadeiro, Fuxo de Can, 29TPG3564, muro de contención, SANT-Bryo 2211. Con nuestra cita se amplía el área de distribución provincial y viene a completar las ya conocidas referencias de Casares-Gil(1932) y Allorge(1934).

Rhynchostegium alopecuroides Brid.

Sierra de Invernadeiro, arroyo de Cernadiño, 29TPG4264, rocas sumergidas, SANTBryo 2212. Nueva localización orensana a añadir a la inicial de Piñor Allorge(1934). Segunda cita provincial.

Rhytidiadelphus squarrossus (Hedw. ) Warnst.

ORENSE: Sierra de Invernadeiro, Vega de Meda, 29TPG3965, terrícola en suelos de bosques, SANT-Bryo 2213. Taxon frecuente en toda Galicia, sin embargo, en la provincia ha sido escasamente citado, Casares-Gil(1915) lo señala en Xinzo de Limia y Rungby(1964) en las cercanías de la capital.

Rhytidiadelphus triquetrus (Hedw. )Warnst.

ORENSE: Sierra de Invernadeiro, Vega de Meda, 29TPG3965, suelo de bosque, SANT-Bryo 2214. Se conoce el taxon en las localidades coruñesas de El Ferrol Colmeiro(1867) y Caaveiro Reinoso(1984) y en las orensanas de Melias y Loña, Luisier(1918).

Thamnobryum alopecurum (Hedw. ) Nieuwl.

ORENSE: Sierra de Invernadeiro, Val de Figueiro, 29TPG3668, talud, SANT-Bryo 2215. Conocemos su presencia en la provincia por la cita de Luisier(1918), desde entonces no se había vuelto a señalar. Segunda cita provincial.

Tortula marginata (B. S. G. )Spruce

ORENSE: Sierra de Invernadeiro, Rucín, 29TPG3768, muro, SANT-Bryo 2216. En la provincia conocido de las cercanías de la capital, Rungby (1964). Segunda cita provincial.

Weissia controversa Hedw. *

ORENSE: Sierra de Invernadeiro, arroyo de Puxo de Mogos, talud, SANT-Bryo 2217. Se conocen pocas referencias del taxon en Galicia, a la inicial de Müller(1854) hay que añadir la de Reinoso y Alvarez(1984).

\section{BIBLIOGRAFÍA}

ALLORGE, P. -1928- Bryotheca iberica. I, Série núm. : 1-50, Espagne: 1-29. Paris.

ALLORGE, P. -1929- Schedae ad Bryothecam Ibericam. II, Série núm. : 51-100, Espagne: 1 27. Paris.

ALLORGE, P. -1934- Notes sur la flore bryologique de la Péninsule Ibérique. IX. Muscinées des provinces du Nord et du Centre de l'Espagne. Rev.Bryol.Lichénol., 7: 249-301. Paris.

CARBALLEIRA, A., R. DEVESA y C. RETUERTO -1983- Bioclimatología de Galicia. Ed. Fund. Barrié de la Maza. La Coruña. 
CASARES-GIL, A. -1915- Enumeración y distribución geográfica de las Muscíneas de la Península Ibérica. Trab. Mus. Nac. Cien. Nat. Ser. Bot., 8: 1-179. Madrid.

CASARES-GIL, A. -1932- Flora Ibérica. Briófitas (segunda parte). Musgos. Mus. Nac. Cien. Nat. Madrid.

CASAS, C. -1972- Brioteca Hispanica 1969. Acta Phytotax. Barc., 6: 16-22.

COLMEIRO, M. -1867- Enumeración de las Criptógamas de España y Portugal.Rev. Progresos Cienc., 16-17: 54-119. Madrid.

COLMEIRO, M. -1889- Enumeración y revisión de las plantas de la península hispanolusitana e islas Baleares con la distribución geográfica de las especies y sus nombres vulgares, tanto nacionales como provinciales (Monocot. y criptógamas)5: 473-571. Madrid.

GARCÍA, C., E. DÍAZ y J. REINOSO -1989- Contribución al estudio de la flora briológica de la cuenca del río Mera (Lugo). Bol. R. Soc. Esp. Hist. Nat. (Sec. Biol.), 84 (3-4): 259271.

LEVIER, R. -1880- Mousses récoltées en 1878 en Espagne et en Portugal. Lausanne.

LUISIER, A. -1918-Fragments de Bryologie Ibérique. 14. Mousses de Galice. Broteria Ser. Bot., 16: 123-142.

MERINO, P. -1916- Contribución a la muscología de la Península Ibérica. Bol. R. Soc. Esp. Hist. Nat., 16: 270-276.

MÜLLER, K. -1854- Bryologische Beitrage zu einer Flore du Pyrenäen, des Nördlichen und der Südlichen Spaniens. Bot. Zeitung, 313-320.

POTIER DE LA VARDE, R. -1945- Liste des spèces du genre Fissidens récoltées dans la Péninsule Ibérique par M. et Mme Allorge. Rev. Bryol. Lichénol., 15: 30-39.

REINOSO, J. -1984- Contribución al conocimiento de la flora briofítica de Galicia. Briófitos de la fraga de Caaveiro (La Coruña). I. Musgos. Lazaroa, 6: 237-243.

REINOSO, J. -1985-Adiciones a la brioflora gallega. Portug. Acta Biol. (B), 14: 155-160.

REINOSO, J. y J. ÁLVAREZ -1984- Estudio briológico de la cuenca del río de Rojos (Santiago). Acta Cient. Compost., 21: 273-284.

RUNGBY, S. -1964- A contribution to the Bryophytic Flore of Spain. Botaniska Notiser, 117: 167-172.

(Aceptado para su publicación en Mayo de 1992)

Dirección de los autores: Departamento de Biología Vegetal (Botánica). Facultad de Biología. Universidad de Santiago de Compostela.

\title{
38. POTENTILLA SUPINA L. (ROSACEAE) EN LA COMUNIDAD VALENCIANA
}

\author{
Juan-José HERRERO-BORGOÑÓN y Manuel B. CRESPO
}

Palabras clave. Corología, Potentilla, Valencia, España.

Durante la realización de un estudio de la flora espontánea de las áreas ajardinadas del viejo cauce del río Turia, a su paso por la ciudad de Valencia, se descubrió una planta perteneciente al género Potentilla, de cuya existencia en la flora valenciana no parecían existir referencias previas. Se trata de $P$. supina L., planta poco frecuente en la Península Ibérica, para la cual se ofrecen seguidamente algunos datos de interés. 